\title{
Certainties and uncertainties in accessing toxicity of non-extractable residues (NER) in soil
}

\author{
Joop Harmsen ${ }^{1}$, Dieter Hennecke ${ }^{2^{*} \mathbb{D}}$, Kerstin Hund-Rinke${ }^{2}$, Joost Lahr ${ }^{1}$ and John Deneer ${ }^{1}$
}

\begin{abstract}
Background: Discussion concerning the bioavailability and ecotoxicological relevance of non-extractable residues (NER) in soil is still ongoing. Is NER formation a detoxification process or a hidden hazard? The use of radiolabelled chemicals enables detection of NER, but the identity of NER is usually unknown. Regulations require clear measurable parameters and the approach of Ortega-Calvo et al. (Environ Sci Technol 49:10255-10264, 2015) defines these.

Results: Following that approach, we studied the fate of three ecotoxic, NER-forming chemicals over a period of 6 months after application to three different soils. Initial ${ }^{14} \mathrm{C}$ experiments showed formation of NER for all chemicals. For the chemical 2,4,6-trinitrotoluene (TNT), NER-formation was reproducible in all soils. We applied a recently standardized method using Tenax ${ }^{\circledR}$ to remove the bioavailable fraction of the chemical at test start and test end. Removing the bioavailable fractions also removed toxicity. Further experiments without radiolabelled TNT clearly showed that the toxicity measured in applied soils was caused by the bioavailable chemical and not by NER.

Conclusions: The tool developed can be used if the fate of the chemical including NER formation is well known and reproducible. The other selected chemicals, cypermethrin and carbendazim, showed unexpected behaviour in ${ }^{14} \mathrm{C}$-fate experiments. The degree of biodegradation was not reproducible for cypermethrin and unexpected losses occurred with carbendazim. This indicated a very large uncertainty when using non-radiolabelled compounds in NER experiments and thus the tool is not suitable in non-radiolabelled experiments.
\end{abstract}

Keywords: NER, Bioavailability, Detoxification, Risk assessment

\section{Background}

The discussion concerning the bioavailability and ecotoxicological relevance of non-extractable residues (NER) in soil is still ongoing [5-7, 22, 24, 28, 32-34]. Is NER formation a detoxification process or should it be considered a hidden hazard? The term non-extractable residues (NER) originates from pesticide regulation where the use of ${ }^{14} \mathrm{C}$-radiolabelled substances is mandatory to determine transformation pathways. The importance of NER, which can be detected only with radiolabelled test substances, was recognized: "What was formerly not detectable suddenly became apparent" [23]. As a consequence

\footnotetext{
*Correspondence: dieter.hennecke@ime.fraunhofer.de

${ }^{2}$ Fraunhofer Institute for Molecular Biology and Applied Ecology, Auf dem Aberg 1, 57392 Schmallenberg, Germany

Full list of author information is available at the end of the article
}

"The concept of persistency or instability of pesticides had to be completely reconsidered" [31].

NER is defined as: 'substances in soils, plants or animals which persist in the matrix after extraction in the form of the parent substance or its metabolites that are indistinguishable from naturally occurring substances. The extraction must not substantially change the substances themselves nor the nature of the matrix' $[8,10]$. In this context 'metabolites' include degradation products of the parent which may show very limited similarity to the parent due to near-complete degradation.

In a review paper, Bariusso et al. [1] reported that pesticides form NER in amounts ranging from a few percent up to $90 \%$. A stimulant for the discussion and research were the two ECETOC reports [3, 4] in which it was suggested to develop methods to assess NER for regulation.
Springer Open

(c) The Author(s) 2019. This article is licensed under a Creative Commons Attribution 4.0 International License, which permits use, sharing, adaptation, distribution and reproduction in any medium or format, as long as you give appropriate credit to the original author(s) and the source, provide a link to the Creative Commons licence, and indicate if changes were made. The images or other third party material in this article are included in the article's Creative Commons licence, unless indicated otherwise in a credit line to the material. If material is not included in the article's Creative Commons licence and your intended use is not permitted by statutory regulation or exceeds the permitted use, you will need to obtain permission directly from the copyright holder. To view a copy of this licence, visit http://creativeco mmons.org/licenses/by/4.0/. 
Typically, in experiments on soils, organic chemicals are extracted from the solid matrix using solvents. Such solvents do not change the chemical or the chemical composition of the soil matrix. However, the physical structure of the matrix might be changed. To extract a chemical, the extractant has to reach the compound (accessibility) and to release the compound (dissolution) [11]. Non-polar organic chemicals dissolve in non-polar solvents like hexane or chloromethanes. Nevertheless, for the extraction of non-polar chemicals from, e.g. soils, non-polar solvents might turn out to be unsuitable because they cannot reach and access the micro-pores. Chemicals present in these pores would be considered non-extractable, though starting extraction with more polar solvents like acetone or methanol will extract more chemical from the matrix. All in all, development of an efficient extraction scheme may be quite difficult. Extraction methods designed to measure the total concentration should include the considerations above and care should be taken that all the chemicals that can be removed are extracted. The chemical remaining is non-extractable and cannot be measured with conventional chemical analytics and consequently it cannot be included in environmental monitoring. In this paper, we are looking for possibilities to supply data that can be used to assess if there is residual toxicity by this 'undetectable' fraction.
During the initiation period of this investigation, 20142016, the implementation of the concept of bioavailability was extensively discussed in scientific meetings. Although bioavailability was already studied for over 20 years [2], it was not implemented in regulation. The 10th SETAC Europe Special Science Symposium, BIOAVAILABILITY OF ORGANIC CHEMICALS (14-15 October 2014 in Brussels), played a very important role for bringing the topic back to regulation. The project team and monitoring team of this project were strongly involved in the organization, presentation, leading and participation in discussions and creation of the resulting paper [26]. NER became part of the developed concept (see Fig. 1). The paper was discussed in several scientific meetings, included in standardization (ISO/TS 16751) [20] and a large number of publications refer to it (73 citations up to December 2019). Therefore, the paper by Ortega-Calvo et al. [26] is considered to be a proper basis for further implementation in risk assessment.

The approach of Ortega-Calvo et al. [26] is based on clearly defined and measurable fractions. The only fraction not measurable is NER, but if all other fractions are measured the remainder represents NER. The approach focusses on the chemical actually present in the soil and pore water and earlier losses (e.g. mineralization, volatilization) are not taken into consideration.

In prospective chemical risk assessment the use of radiolabelled test substances is obligatory, enabling the

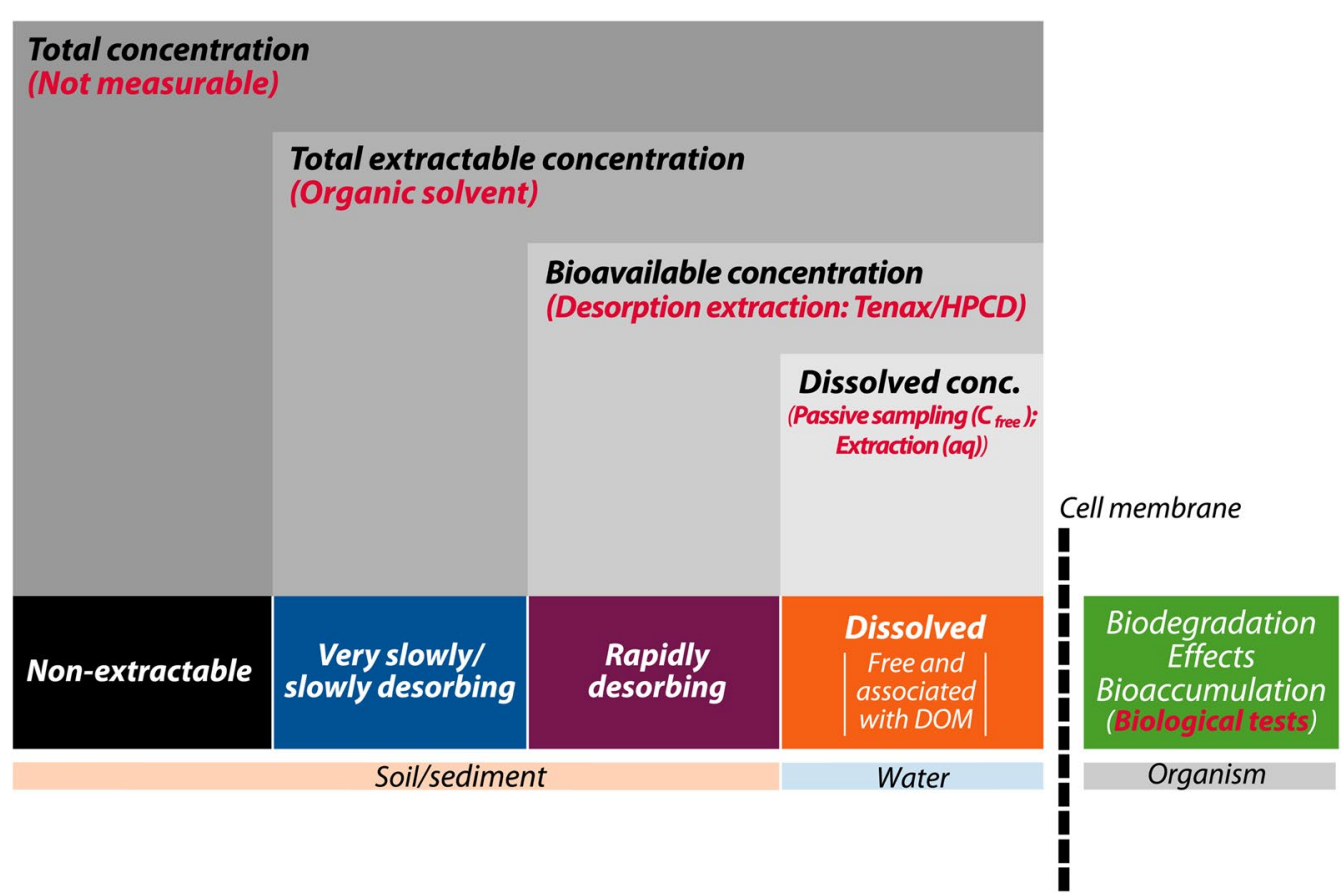

Fig. 1 Bioavailability and NER according to Ortega-Calvo et al. [26] 
quantification of NER. However, the possible occurrence of NER makes the risk assessment in prospective regulation more difficult. Many physical and chemical processes were identified in the past that can lead to a more or less irreversible sorption or entrapment of chemicals in the solid matrix in soils and sediments [22]. Detection of the radiolabel does not mean that the chemical is still present. Even after degradation of the chemical the labelled carbon might still be present in the matrix, e.g. incorporated into a biological molecule as a product of the metabolism by microorganisms. Kästner et al. [22] developed a classification approach for NER. According to specific extraction steps the total amount of NER is the sum of:

- strongly adsorbed, sequestered or entrapped residues (type I NER),

- covalently bound residues (type II NER),

- natural biogenic molecules (type III or biogenic NER).

Both type I and type II NER are derived from either the parent chemical or from transformation or degradation products, whereas type III NER are derived from biotic/biological degradation or the anabolic use of the chemical.

Methods are suggested to identify the presence of the different NER types, silylation [28] to distinguish between type I and type II NER and analysis of radiolabelled amino acids after acidic hydrolysis for biogenic NER (Type III NER). However, methods are still under development and currently type I and type II NER cannot be determined as pure fractions. If type III NER are present in the matrix, they will stay either in the solid matrix after silylation and as such contribute to the type II NER or they become dissolved and add to type I NER. Therefore, computer models have been developed, to estimate biogenic NER based on the measured mineralization of the chemical (MTB method, [29]). Apart from the approach according to Kästner et al. [22], other concepts were developed (e.g. [9]) which are principally similar but have some procedural differences. However, independent of which methodology for NER characterization is used, radiolabelled chemicals are necessary.

Important in the approach of Kästner et al. [22] is the inclusion of kinetics in the definition: 'Potential to become released' in type I NER and 'very low remobilization rates' in type II NER. Scientifically this is correct and may lead to a better understanding of NER. On a geological scale, all chemicals become available, but kinetics should be considered within the time frame of a risk assessment. A very low remobilization rate does not automatically mean that the chemical is non-extractable. polycyclic aromatic hydrocarbons (PAHs) are used as examples for NER [30]. The residual fraction present after bioremediation (i.e. biodegradation of the bioavailable fraction) can be identified as type II NER. In a 25-year study Harmsen and Rietra [13] have shown that the residual part of PAHs in soil degrade at a very slow rate (half-life time 11.5 years), which is driven by the very slow desorption of PAHs. Moreover, the PAHs were still extractable, which shows that extra terms like very low rates, can be a contradiction to the meaning of non-extractable residue. In order to avoid confusion, we think that in the future a further discussion of definitions is necessary to prevent the existence of different "Scientific schools". Apparent differences in definitions are a big obstacle when implementing in risk assessment.

As mentioned, the use of radiolabelled chemicals is needed to show the presence of NER, but this is not normally feasible in experiments aiming to demonstrate toxicity caused by the chemical and/or residual toxicity of NER. If non-radiolabelled chemicals are used in ecotoxicological experiments NER is then not measurable. In this paper, we present some findings obtained during the development of a tool to assess ecotoxicity of NER formed from chemicals with known ecotoxicity.

Taking into account all the uncertainties described above, we were guided by the following objectives:

1. Understanding of NER-formation by testing with radiolabelled chemicals.

2. Translation of the results into a testing tool to be implemented in risk assessment. The tool had to be transparent to risk assessors with focus on measurable parameters.

3. Application of the developed know-how in a general applicable testing tool with non-labelled chemicals.

\section{Materials and methods}

\section{Selection of test substances}

The first step in the project was the selection of suitable test chemicals. The chemicals should: (1) form significant amounts of NER in soils within an experimental period of 6 months; (2) have a high toxicity to terrestrial test organisms; (3) be available as ${ }^{14} \mathrm{C}$-radiolabelled substance at reasonable cost and (4) be non-ionizable so as not to complicate the experimental part of the study due to the formation of charged forms of the chemical.

As a result of an extensive literature survey, it turned out that selection of the test chemicals was much more complex than expected. The combination of NER-formation and high terrestrial toxicity is not very common. A refined selection was derived from NER formation and only included chemicals that had shown relevant 
terrestrial toxicity. From this list 2,4,6-trinitrotoluene (TNT), cypermethrin and carbendazim were selected to be used for pre-tests. Details on structure, position of radiolabelling, providers and some physico-chemical properties are given in Table 1. Methods used and all collected results during the study of 3 years are described in more detail in Harmsen et al. [12].

\section{Soil incubation and extraction}

\section{Experimental setup for determination of the fate of the test} substances

The basic idea behind the testing tool derived is to test the soil with an ecotoxicological assay before and after removal of the bioavailable fraction and to apply the test just after spiking and after aging, i.e. after NER is formed.

Using solvents to remove the bioavailable part and subsequently performing ecotoxicological tests with the extracted soil is not possible because the solvents will cause toxicity.

In the approach of Ortega-Calvo et al. [26] fractions of chemicals are well defined, clear and measurable. The only fraction not measurable directly without radiolabelled chemical is NER, but this can be considered and quantified as a residual fraction if all others are measured in the same soil sample and the fate of the chemical is well known. The methods for determination of the bioavailable fraction have a mechanistic principle and measure the amount of the chemical in the water phase (actual) and the amount that rapidly desorbs to the water phase (potential). The fraction of the chemical determined with an organic extraction desorbs also slowly or very slowly, but due to the very slow release concentrations are too low to cause toxic effects. The fractions considered are:

- Chemical present in the water phase, actually bioavailable $\left(\mathrm{CaCl}_{2}\right.$-extraction);

- A potentially bioavailable fraction (Tenax $20 \mathrm{~h}$, ISO TS-16751 [20]);

- The extractable residue, measured in organic solvent extract after Tenax $20 \mathrm{~h}$;

- NER as non-measurable and non-bioavailable remaining fraction after extraction.

Three different German reference soils (http://www. refesol.de) were selected for the experiments, based on differences in their properties. Clay content in soil 01-A, $02-\mathrm{A}$ and $03-\mathrm{G}$ was $6.1,15.7$ and $22.0 \%$, respectively. Organic carbon content was $0.80,0.92$ and $2.46 \%$ in the three soils, respectively.

The amount of organic solvent (acetone) used for spiking was limited to $0.6 \% \mathrm{w} / \mathrm{w}$ in order to minimize the risk of ecotoxicological effects caused by the solvent or by increased oxygen consumption due to solvent degradation. Initial concentrations of the test substances in the experiments were $120 \mathrm{mg} / \mathrm{kg}$ soil dry matter, which is very high compared to environmental concentrations and thus somewhat artificial. These high concentrations were selected in order to ensure sufficiently high concentrations of parent compound, metabolites and NER after 6 months of aging, resulting in toxic effects despite detoxification through degradation and mineralization.

The spiked soil was incubated under standard aerobic conditions at $20{ }^{\circ} \mathrm{C}$ in the dark. In the first experiment

Table 1 Details on ${ }^{14} \mathrm{C}$-radiolabelled substances used for the project

Substance name
2,4,6-TNT
IUPAC: 2-methyl-1,3,5-trinitrobenzene
CAS-No 118-96-7


with ${ }^{14} \mathrm{C}$-radiolabelled substances (hereafter "mass balance experiment"), the overall fate and mass balance of each substance was followed by monthly sampling (triplicates) and quantification of the extractable radioactivity (liquid scintillation counting (LSC) of organic extract), the mineralization $\left({ }^{14} \mathrm{CO}_{2}\right.$-trapping) and the non-extractable radioactivity (LSC after combustion of extracted residue). Since for cypermethrin in the first experiment toxicological effects on the soil were still at a maximum at the end of the incubation period, an additional experiment was performed at an initial concentration of $5 \mathrm{mg} /$ $\mathrm{kg}$, which is still high compared to environmental concentrations. NER-formation and mineralization were monitored and compared to the $120 \mathrm{mg} / \mathrm{kg}$ experiment.

In the second ${ }^{14} \mathrm{C}$-radiolabelled experiment (hereafter "distribution experiment"), the distribution of the chemical over fractions representing different levels of bioavailability (see Fig. 1) was measured directly after spiking and after a 6-month aging period. Apart from the determination of the distribution of the chemical in the matrix, these experiments were intended to provide soil material containing only NER for further ecotoxicological testing. Therefore, no ${ }^{14} \mathrm{CO}_{2}$-trapping was performed in the distribution experiment. Toxicity after spiking and aging was tested in a parallel non-radiolabelled second experiment using non-radiolabelled chemicals. The following sequential extractions were applied, see also "Development of a testing tool":

- Extraction with $0.01 \mathrm{M} \mathrm{CaCl}_{2}$. Actually bioavailable fraction (Houba et al. [14]);

- Tenax extraction over $20 \mathrm{~h}, \mathrm{ISO} / \mathrm{TS} 16751$ [20]. Potentially bioavailable or rapidly desorbing fraction;

- Tenax extraction over 1 week, slowly desorbing fraction; provision of aliquot from extracted soil residue for ecotoxicological testing of soil;

- Soil aliquot: extraction of the total extractable amount using organic solvent, preferably standardized method (e.g. ISO 11916 [17] for TNT). This part might desorb, too, but very slow;

- Soil aliquot: extraction using pressurized liquid extraction (PLE).

\section{Experiments with soil for subsequent ecotoxicological testing} Based on the ${ }^{14} \mathrm{C}$-experiments described above (see "Experimental setup for determination of the fate of the test substances") a testing tool for NER assessment was developed. The tool itself does not require the use of ${ }^{14} \mathrm{C}$-radiolabelled substances, but the information about substance behaviour determined in the ${ }^{14} \mathrm{C}$-experiments was needed to avoid incorrect conclusions. Reasonable and reproducible results of the ${ }^{14} \mathrm{C}$-experiments are the only proof for NER formation in the subsequent experiments when developing the testing tool.

Basically the application rate and the procedure and incubation of soils in the non-radiolabelled test were similar to those described in "Experimental setup for determination of the fate of the test substances" for the ${ }^{14} \mathrm{C}$-radiolabelled experiments. The experiments incorporating fractionation of different levels of bioavailability were only performed with the non-radiolabelled test substance. The 1 week Tenax extraction was omitted in this experiment. Further details are described in "Development of a testing tool".

\section{Radio-TLC chemical analysis}

In order to differentiate between the parent test substances and potential transformation products, all extracts from the ${ }^{14} \mathrm{C}$-distribution experiments were analysed by radio-TLC (thin layer chromatography). Aliquots of extracts were applied to silica gel-coated TLCplates and after evaporation of the solvent the plates were eluted using a defined solvent mixture in a glass TLCchamber. For detection, the plates were exposed to a radioactivity-sensitive film. The film was read by a Typhoon FLA 700 plate reader. Software (AIDA) converts the plate information into a chromatogram and the distribution of the radioactivity read from the plate allows a quantitative evaluation of test substance and metabolite distribution. Details of the analyses are provided in Table 2.

\section{Ecotoxicity tests}

Using toxicity tests with short incubation periods, hours or days instead of weeks or months, the NER concentration can be assumed to be relatively constant over the duration of the test. If longer, chronic, tests had been used, the chemicals would have changed from bioavailable to NER over the course of the toxicity test. This is especially true for freshly spiked samples and there is uncertainty as to what is measured: toxicity just after spiking or toxicity at the end of the toxicity experiment. After several months a more stable state is obtained, but

Table 2 Radio-TLC-methods applied

\begin{tabular}{lcc}
\hline Test chemical & Solvent mixture $\mathbf{v} / \mathbf{v}$ in $\mathbf{~ m l}$ & TLC-phase \\
\hline TNT & Acetonitrile/water:70/30, v/v & Merck TLC \\
& & Silica gel \\
& & 60, RP18, \\
& & F254 \\
& & (RP18) \\
Cypermethrin & n-Hexane/acetone: 80/20, v/v & Merck TLC \\
Carbendazim & n-Hexane/ethylacetate/trichlorometh- & Silica gel \\
& ane/tetrahydrofuran/acetic acid: & 60, F254 \\
& 30/30/30/6/2, v/v/v/v/v & (KG60) \\
& & \\
\hline
\end{tabular}


a correct evaluation of (NER-) toxicity by comparing toxicity after spiking and after NER-formation would not have been possible. Consequently, only acute tests were used in our experiments. The results of these tests provide more accurate information on the actual bioavailability of the contaminants at the date of sampling. Instead of the more common earthworm reproduction test which needs an 8-week testing period, we applied the earthworm avoidance test in soil [19] with only a 2-day testing period. For this test, adult earthworms are exposed at the same time to the control soil and a soil containing the test substance. These soils are filled into the two sections of the same vessel and the earthworms can choose between the test soil and the control soil. After an incubation period of 2 days, the number of worms is determined in both sections of the vessels and the percentage of avoidance is calculated. We further used the Daphnia test [25] and luminescence bacteria test [15] for eluates. For the test with daphnids, young organisms, aged less than $24 \mathrm{~h}$ at the start of the test, are exposed to the eluates at a range of concentrations for a period of 2 days. Immobilization is recorded after $24 \mathrm{~h}$ and $48 \mathrm{~h}$ and the percentage of immobilization is calculated. For the second test, the organisms belong to the luminescent bacteria. In the presence of toxic substances the light emission is reduced. The bacteria (Vibrio fischeri) are exposed to several concentrations of the eluate for a period of up to $30 \mathrm{~min}$ and percent reduction of the luminescence is calculated. The tests are acute tests with short incubation periods. A chronic test can only be applied when a stable situation is obtained regarding NER formation. In addition, currently not much is known about NER modification processes which might also have an effect on NER toxicity. Therefore, it was decided to use acute tests to get some kind of a snapshot on actual NER toxicity.

For every test chemical at least one terrestrial test and one test with soil eluates were selected. The selection was based on the sensitivity of the test organisms to the test compound known from the literature [12] and our pretests. Toxicity in the spiked soil was measured immediately after spiking and after 6 months of incubation.

The ammonium oxidation test [16] proved to be unsuitable for the purpose of the research described. The test principle of the soil microflora test differs from the principles of the earthworm and the aquatic tests. In the latter tests the test organisms are added to the test soils. However, in the soil microflora tests, the activity of the natural microbial community already present is determined. The recovery of the microflora during the incubation period proved to be too low to assess the bioavailable amounts of contaminants correctly. The combinations of test chemical and ecotoxicological tests shown in Table 3 were considered suitable.

$\begin{aligned} & \text { Table } 3 \\
& \text { Toxicity } \\
& \text { of the testing tool using radiolabelled chemicals }\end{aligned}$
\begin{tabular}{lll} 
Chemical & Medium & Test applied \\
\hline TNT & Soil & Avoidance test \\
& Soil eluate & Luminescent bacteria test \\
$\begin{array}{l}\text { Cypermethrin and } \\
\text { carbendazim }\end{array}$ & Soil & Avoidance test \\
& Soil eluate & Daphnia test \\
\hline
\end{tabular}

\section{Development of a testing tool}

Based on the first two objectives, a tool was developed to assess if NER present in soil could be responsible for residual toxicity. The tool is based on the principle shown in Fig. 1. Parameters should be measurable and both sides of the cell membrane from Fig. 1 have to be considered. The tool was applied to non-radiolabelled 2,4,6-TNT, because the radiolabelled experiments (see "Experimental setup for determination of the fate of the test substances") showed significant and reproducible formation of NER in soil over time. Basically, if the fate of a substance is well known, then there is no need to work with ${ }^{14} \mathrm{C}$-radiolabelled substances. However, one should consider, that in this case there is no direct proof of the amount of NER tested in subsequent toxicity tests, since NER cannot be measured in a non-radiolabelled experiment. All uncertainties have to be considered when interpreting the results of the experiment.

For chemical analysis and toxicity testing different extraction procedures, carried out sequentially, were used. The definitions [18, 26] of the extracted fractions [27] are explained below and they are applied, for example in Figs. 3 and 5:

Actually bioavailable: substance concentration in the water phase, in which it can be measured using extractions with $0.01 \mathrm{M} \mathrm{CaCl}_{2}$;

Potentially bioavailable: substance fraction normally in equilibrium with the water phase. Measured using ISO/TS 16751 [20]: we used Tenax to collect the fraction of potentially bioavailable chemical from the water phase over $20 \mathrm{~h}$. The Tenax was extracted and the concentration of the substance was measured in the extract. The amount recovered from the Tenax represents the potentially bioavailable fraction. For practical reasons the extraction with Tenax for 1 week, which enables the measurement of the slowly desorbing fraction (see Fig. 1), was omitted. This fraction is considered to be part of the non-bioavailable fraction. In contradiction with the experiments for determining the fate of substances process ("Experimental setup for determination of the fate of the test substances"), both bioavailabilities were measured in parallel and not sequential. 
Residue: the total extractable amount after 20-h Tenax extraction was determined by extraction with a suitable solvent, preferably following a standardized method. In our study it contains both, the slowly desorbing fraction of Tenax 1 week and the very slowly desorbing fraction. Following ISO 11916 [17], acetonitrile was used to extract 2,4,6-TNT.

NER: was considered, but was not measurable in these tests, as we used non-radiolabelled test substances. However, from the ${ }^{14} \mathrm{C}$-radiolabelled tests the order of magnitude of NER formed from 2,4,6-TNT during incubation in the same soil under the same standard conditions was known. As the other measurable fractions were not so different between the ${ }^{14} \mathrm{C}$-radiolabelled and nonradiolabelled experiments, NER could be estimated with confidence.

Generally, if the fate of the chemical is understood from radiolabelled experiments and repeatable, NER can be estimated in non-radiolabelled experiments. However, to quantify NER simply as $100 \%$ minus the extractable amount is very risky and might lead to incorrect results, as experiments with ${ }^{14} \mathrm{C}$ carbendazim demonstrated.

Measurement of the bioavailability and ecotoxicity of the substance representing NER was performed using the organisms described in the test guidelines (avoidance test with earthworms Eisenia fetida, ISO 17512-1 [19]; immobilization test with daphnids Daphnia magna, OECD TG 202 [25]; luminescence inhibition test with Vibrio fischeri, ISO 11348 [15]). For those tests it is necessary that the chemical is toxic to the selected organism at the NER "concentration" (luminescent bacteria test for TNT). Toxicity tests were conducted using freshly spiked soil and aged soil, 5 months for soil 01 -A and 7 months for the other 2 soils. In order to conduct the ecotoxicological tests simultaneously with the same batch of test organisms, new soil was freshly spiked a day before the aged soil was investigated and tested for toxicity together with the aged soil.

\section{Results and discussion}

\section{Understanding of NER formation and NER toxicity} NER formation of ${ }^{14} \mathrm{C}-\mathrm{TNT}$

The NER formation kinetics, as measured in the mass balance experiment, are presented in Fig. 2. A high rate of formation of NER was observed in each of the three test soils.

Mineralization, measured as formation of ${ }^{14} \mathrm{CO}_{2}$, was limited to a maximum of $7 \%$ within 6 months. Extraction with acetonitrile was applied to determine the extractable amount (see [17]). The subsequent pressurized liquid extraction (PLE) yielded an extra 5\%. This is the result of residual solvent in the extracted soil rather than the

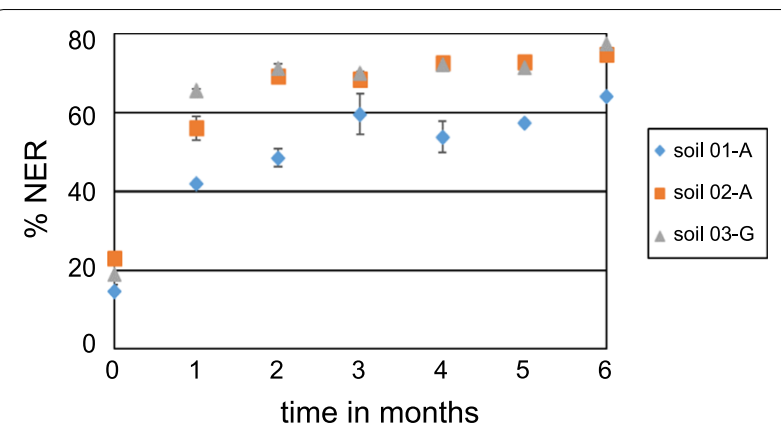

Fig. 2 NER formation of TNT in three soils, experiment 1 (mean of triplicate sampling)

stronger extraction power by PLE. The mass balance was mostly above $95 \%$ of the applied radioactivity.

The distribution experiment with ${ }^{14} \mathrm{C}$-labelled test substance was conducted to measure both the distribution over different fractions according to Ortega-Calvo et al. [26] and the toxicity of the soil. Mass balance was not considered in the distribution experiment and therefore no ${ }^{14} \mathrm{CO}_{2}$ was trapped and measured as this was not relevant for NER toxicity determination. Accordingly the "total recovery" shown in Table 4 does not represent the radioactive mass balance of the experiment. Mineralization was determined in the mass balance experiment described above. Also, both were conducted directly after spiking and after 6 months of aging. In this experiment the extraction was performed sequentially (see "Experimental setup for determination of the fate of the test substances"). Results are summarized in Table 4.

Results in Table 4 show that most of the TNT in the soils shortly after spiking is present as bioavailable TNT as the amount in the water phases are high. This is explained by the relatively high solubility of TNT in water (approx. $100 \mathrm{mg} / \mathrm{l}$ [21]). The NER-formation found in the distribution experiment is lower than observed in the mass balance experiment.

Radio-TLC analysis showed that the percentage of parent TNT in the Tenax extracts changed from 29 to $48 \%$ $\mathrm{AR}$ at test start to approximately $3-10 \% \mathrm{AR}$ at test end.

\section{Toxicity of TNT-NER}

The experiment presented in Table 4 had a parallel experiment using non-radiolabelled TNT due to the high amounts of soil required for the earthworm avoidance test. Luminescent inhibition needs less soil and could be measured using soil material from the ${ }^{14} \mathrm{C}$-radiolabelled experiment. Toxicity of the soil directly after spiking and after aging was measured with the earthworm avoidance assay and luminescence inhibition. Together with the amounts extracted in the sequential extractions, the 
Table 4 Presence of radioactivity in the different extracts of the sequential extraction (applied radioactivity 5.1 kBq ${ }^{14} \mathrm{C}$ TNT per $\mathrm{g}$ soil corresponding to $120 \mathrm{mg}$ 2,4,6-TNT per $\mathrm{kg}$ soil $\mathrm{dm}$ )

\begin{tabular}{|c|c|c|c|c|c|c|c|c|c|}
\hline \multirow[t]{2}{*}{ Soil } & \multirow[t]{2}{*}{$0.01 \mathrm{M} \mathrm{CaCl}_{2}$} & \multicolumn{2}{|c|}{$\operatorname{Tenax}_{20}$} & \multicolumn{2}{|c|}{ Tenax $_{1 \text { week }}$} & \multirow{2}{*}{$\begin{array}{l}\text { Acetonitrile } \\
\text { shaking }\end{array}$} & \multirow[t]{2}{*}{ PLE } & \multirow[t]{2}{*}{ NER } & \multirow[t]{2}{*}{ Total recovery } \\
\hline & & Water & $\overline{\text { Tenax }}$ & Water & $\overline{\text { Tenax }}$ & & & & \\
\hline \multicolumn{10}{|c|}{ Test start } \\
\hline 01-A & $47.6 \%$ & $4.6 \%$ & $28.0 \%$ & $1.5 \%$ & $5.2 \%$ & $2.3 \%$ & $0.2 \%$ & $2.8 \%$ & $92.2 \%$ \\
\hline $02-A$ & $40.3 \%$ & $4.8 \%$ & $36.9 \%$ & $2.5 \%$ & $3.8 \%$ & $2.8 \%$ & $0.6 \%$ & $9.3 \%$ & $101.0 \%$ \\
\hline O3-G & $23.5 \%$ & $4.9 \%$ & $44.7 \%$ & $3.5 \%$ & $5.6 \%$ & $3.6 \%$ & $0.8 \%$ & $13.4 \%$ & $100.0 \%$ \\
\hline \multicolumn{10}{|c|}{$\mathrm{T}=6$ months } \\
\hline $01-A$ & $6.1 \%$ & $4.3 \%$ & $9.3 \%$ & $6.6 \%$ & $5.4 \%$ & $3.7 \%$ & n.d. & $50.4 \%$ & $85.8 \%$ \\
\hline $02-A$ & $3.4 \%$ & $3.5 \%$ & $7.1 \%$ & $3.7 \%$ & $2.4 \%$ & $6.2 \%$ & & $54.1 \%$ & $80.4 \%$ \\
\hline $03-G$ & $1.3 \%$ & $2.4 \%$ & $5.7 \%$ & $2.7 \%$ & $2.9 \%$ & $5.8 \%$ & & $63.0 \%$ & $83.8 \%$ \\
\hline
\end{tabular}

results are presented in Fig. 3. The change from bioavailable TNT to NER in 6 months corresponds with a strong reduction of toxicity as measured by both earthworm avoidance and luminescence inhibition. Only in soil 03-G residual earthworm avoidance was still present after 6 months.

\section{NER formation of ${ }^{14} \mathrm{C}$-cypermethrin}

In the mass balance experiment, 16, 22 and $38 \%$ of the applied radioactivity was identified as NER after 6 month of aging in soils 01-A, 02-A and 03-G, respectively. Only 1.9, 2.9 and 4\%, respectively, of added cypermethrin was mineralized. In line with this low mineralization, incorporation of radiolabelled ${ }^{14} \mathrm{C}$ in biomass should be low [29] and NER in this experiment is considered more likely to be entrapped parent compound or a breakdown product.

The results of the distribution experiments are shown in Table 5 . The water solubility of cypermethrin is very low ( $9 \mu \mathrm{g} / \mathrm{l}$, IUPAC database), which makes the water phase less important for the mass balance. The concentration in the $\operatorname{Tenax}_{20}$ extract was very high at test start, indicating that most of the cypermethrin applied would be bioavailable to organisms. Even in the Tenax ${ }_{1 \text { week }}$, considerable concentrations were still measured. This demonstrates the good performance of the Tenax extraction and indicates orders of magnitude more cypermethrin bioavailable compared to the $0.01 \mathrm{M} \mathrm{CaCl}_{2}$ extraction. Almost no NER was found immediately after spiking.

After 6 months, the amount of radioactivity extracted in $\operatorname{Tenax}_{20}$ had decreased. At the same time, the slowly bioavailable ( $\left.\operatorname{Tenax}_{1 \text { week }}\right)$ and the residue (acetonitrile) amount had increased, and significant differences were observed between the soils. TLC analysis showed that radioactivity in all extracts was due to parent cypermethrin only.

The largest difference compared to the mass balance experiment were the significantly lower amounts of NER detected. NER in the distribution experiment was lower by a factor of 5-10 after 6 months compared to the mass balance experiment. Further, the results do not match with data for cypermethrin found in the literature survey.
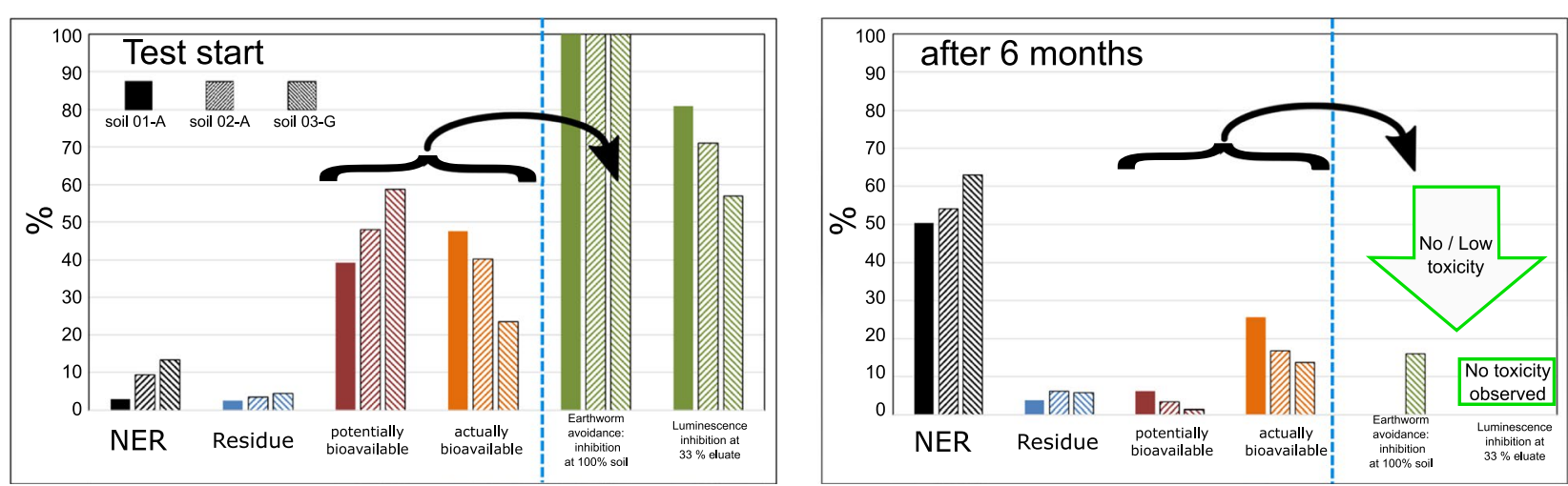

Fig. 3 Results of test with TNT in the three test soils. NER, actually bioavailable, residue and potentially bioavailable expressed as percentage of recovered radioactivity, toxicity as percent inhibition (earthworm avoidance, 100\% test soil; luminescence inhibition, 33\% soil eluate) 
Table 5 Presence of radioactivity in the different extracts of the sequential extraction (applied radioactivity $4.2 \mathrm{kBq}$ ${ }^{14} \mathrm{C}$-cypermethrin per $\mathrm{g}$ soil $\mathrm{dm}$ corresponding to $120 \mathrm{mg}$ cypermethrin per $\mathrm{kg}$ soil $\mathrm{dm}$ )

\begin{tabular}{|c|c|c|c|c|c|c|c|c|c|}
\hline \multirow[t]{2}{*}{ Soil } & \multirow[t]{2}{*}{$0.01 \mathrm{~m} \mathrm{CaCl}_{2}$} & \multicolumn{2}{|c|}{ Tenax $_{20}$} & \multicolumn{2}{|c|}{ Tenax $_{1 \text { week }}$} & \multirow{2}{*}{$\begin{array}{l}\text { Acetonitrile } \\
\text { shaking }\end{array}$} & \multirow[t]{2}{*}{ PLE } & \multirow[t]{2}{*}{ NER } & \multirow[t]{2}{*}{ Total recovery } \\
\hline & & Water & Tenax & Water & Tenax & & & & \\
\hline \multicolumn{10}{|c|}{ Test start } \\
\hline $01-A$ & $0.1 \%$ & $0.3 \%$ & $41.5 \%$ & $0.4 \%$ & $22.5 \%$ & $17.6 \%$ & $1.0 \%$ & $0.3 \%$ & 83.7 \\
\hline $02-A$ & $0.2 \%$ & $0.3 \%$ & $80.6 \%$ & $0.1 \%$ & $5.9 \%$ & $4.1 \%$ & $0.8 \%$ & $0.3 \%$ & 92.3 \\
\hline $03-G$ & $0.2 \%$ & $0.5 \%$ & $42.0 \%$ & $0.3 \%$ & $26.4 \%$ & $21.3 \%$ & $2.9 \%$ & $0.5 \%$ & 94.1 \\
\hline \multicolumn{10}{|c|}{$\mathrm{T}=6$ months } \\
\hline $01-A$ & $0.8 \%$ & $0.0 \%$ & $15.6 \%$ & $0.7 \%$ & $26.5 \%$ & $30.7 \%$ & $1.2 \%$ & $1.4 \%$ & $76.9 \%$ \\
\hline $02-A$ & $0.6 \%$ & $0.0 \%$ & $16.5 \%$ & $0.8 \%$ & $34.0 \%$ & $34.7 \%$ & $2.7 \%$ & $2.3 \%$ & $91.6 \%$ \\
\hline $03-G$ & $2.0 \%$ & $0.4 \%$ & $13.7 \%$ & $0.5 \%$ & $17.5 \%$ & $31.8 \%$ & $5.4 \%$ & $6.4 \%$ & $77.7 \%$ \\
\hline
\end{tabular}

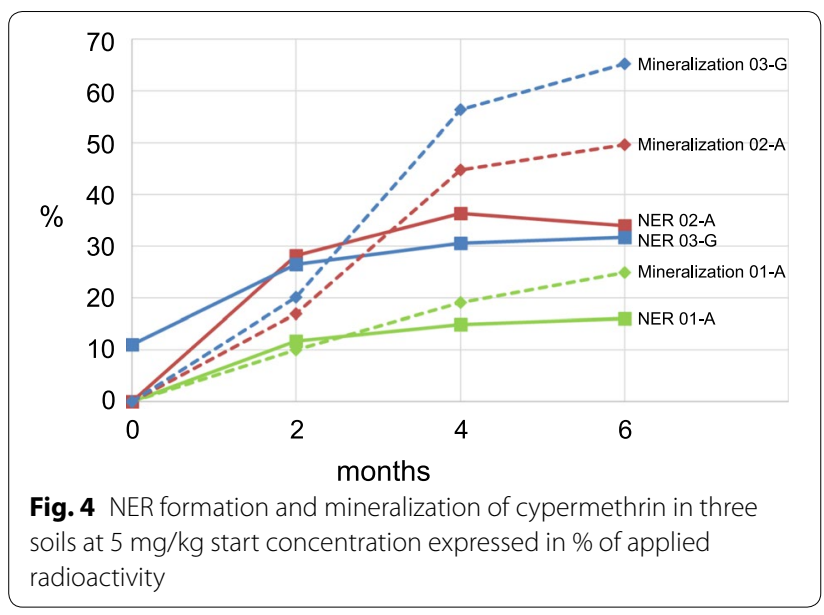

Experimental errors can be excluded as the NER determination is one of the most robust measurements in the entire project and the mass balance, at least for soil 02-A, was above $90 \%$. The difference between NER results in the two experiments cannot be explained.

The high initial concentration of cypermethrin most likely caused lethal effects for many soil organisms. In the experiment using lower $(5 \mathrm{mg} / \mathrm{kg})$ initial concentrations enhanced (approx. 15 times) mineralization was observed (Fig. 4) compared to the mass balance experiment using $120 \mathrm{mg} / \mathrm{kg}$.

\section{Toxicity of cypermethrin NER}

Toxicity tests were performed using the sensitive earthworm avoidance and Daphnia tests. The luminescence inhibition test was less sensitive for cypermethrin and not applied. The results of the toxicity tests with $120 \mathrm{mg} /$ $\mathrm{kg}$ cypermethrin are given in Table 7 and showed that high toxicity was present, both at test start and test end. Solubility of cypermethrin is low anyway, but NER formation did not reduce the already low concentration in the water phase. Consequently, the soil should show the same toxicity which was confirmed by the tests.

\section{NER formation of ${ }^{14} \mathrm{C}$-carbendazim}

In the carbendazim mass balance experiment, the total recovery dropped during the 6-month aging period to 38,56 and $75 \%$ in the three soils $01-\mathrm{A}, 02-\mathrm{A}$ and $03-\mathrm{G}$, respectively. NER formation occurred mostly in the first 2 months and was 27,32 and $56 \%$, respectively, after 6 months of aging. Formation of ${ }^{14} \mathrm{CO}_{2}$ was only moderate $(9,23$ and $8 \%)$.

In the distribution experiment losses of radioactivity were much higher. Although the recovery at test start was still good, it decreased after 6 months to only 7,15 and $36 \%$ of the initially applied radioactivity (see Table 6). Most of that material was present as NER, but the extractable fraction almost disappeared entirely (no single extract $>1 \%$ and none of them only parent carbendazim). Losses were unexpected and we assume that it might be due to volatilization of a degradation product other than ${ }^{14} \mathrm{CO}_{2}$ because, regardless of the ongoing process, the radioactivity would have been recovered if it had still been present in the system. Since the focus of the project was on NER formation rather than metabolism or trapping of volatiles, the experimental setup unfortunately was not appropriate to provide data to explain this observation.

\section{Toxicity of carbendazim NER}

Aging caused a high reduction of toxicity (Table 7) which can be explained by the combined effect of NER-formation and other losses as described in "NER formation of ${ }^{14} \mathrm{C}$-carbendazim". However, due to the high uncertainty in quantifying both degradation and NER formation, the importance of NER for toxicity cannot be established from these experiments. 
Table 6 Presence of radioactivity in the different extracts of the sequential extraction (applied radioactivity $4.8 \mathrm{kBq}$ ${ }^{14} \mathrm{C}$-carbendazim per $\mathrm{g}$ soil $\mathrm{dm}$ corresponding to $120 \mathrm{mg}$ carbendazim per $\mathrm{kg}$ soil $\mathrm{dm}$ )

\begin{tabular}{|c|c|c|c|c|c|c|c|c|c|}
\hline \multirow[t]{2}{*}{ Soil } & \multirow[t]{2}{*}{$0.01 \mathrm{M} \mathrm{CaCl}_{2}$} & \multicolumn{2}{|c|}{$\operatorname{Tenax}_{20}$} & \multicolumn{2}{|c|}{$\operatorname{Tenax}_{1 \text { week }}$} & \multirow{2}{*}{$\begin{array}{l}\text { Methanol } \\
\text { shaking }\end{array}$} & \multirow[t]{2}{*}{ ASE } & \multirow[t]{2}{*}{ NER } & \multirow[t]{2}{*}{ Total recovery } \\
\hline & & Water & Tenax & Water & Tenax & & & & \\
\hline \multicolumn{10}{|c|}{ Test start } \\
\hline 01-A & $19.2 \%$ & $5.5 \%$ & $45.3 \%$ & $0.3 \%$ & $8.7 \%$ & $0.9 \%$ & $0.3 \%$ & $0.6 \%$ & $80.8 \%$ \\
\hline $02-A$ & $17.3 \%$ & $4.7 \%$ & $71.7 \%$ & $0.5 \%$ & $2.3 \%$ & $0.5 \%$ & $0.2 \%$ & $0.7 \%$ & $97.9 \%$ \\
\hline $03-G$ & $10.8 \%$ & $12.2 \%$ & $69.4 \%$ & $0.2 \%$ & $6.3 \%$ & $1.3 \%$ & $0.8 \%$ & $1.3 \%$ & $102.3 \%$ \\
\hline \multicolumn{10}{|c|}{$\mathrm{T}=6$ months } \\
\hline $01-A$ & $0.4 \%$ & $0.7 \%$ & $0.2 \%$ & $0.8 \%$ & $0.2 \%$ & $0.4 \%$ & $0.5 \%$ & $4.2 \%$ & $7.4 \%$ \\
\hline $02-A$ & $0.2 \%$ & $0.3 \%$ & $0.1 \%$ & $0.5 \%$ & $0.1 \%$ & $0.5 \%$ & $0.7 \%$ & $12.2 \%$ & $14.6 \%$ \\
\hline 03-G & $0.9 \%$ & $0.9 \%$ & $0.1 \%$ & $1.0 \%$ & $0.5 \%$ & $0.5 \%$ & $1.9 \%$ & $30.3 \%$ & $36.1 \%$ \\
\hline
\end{tabular}

\section{Lessons learned from ${ }^{14} \mathrm{C}$ experiments}

Instead of using a toxic solvent, Tenax was used to remove the bioavailable fraction. Tenax is a solid phase and can be easily separated from water and soil. Tenax did not have an effect on the bioassay with luminescent bacteria as described in this section. However, care must be taken even after Tenax extraction as not all ecotoxicological test systems work properly any more (e.g. ammonium oxidation, see "Ecotoxicity tests").

Tenax extraction might not be necessary if the soil analysis shows that the substance is already degraded during aging and only NER from that substance is present in the soil.

From a practical standpoint, only limited amounts $(<200 \mathrm{~g})$ of soil can be extracted using Tenax, implying limited availability of soil containing NER for ecotoxicity testing. This is important, e.g. for earthworm testing, where much larger amounts of soil are needed.

For further development of the testing tool, only 2,4,6TNT seemed to be suitable as the fate of the other two

Table 7 Effect of cypermethrin and carbendazim on earthworms and daphnids

\begin{tabular}{|c|c|c|c|c|}
\hline \multirow[t]{2}{*}{ Soil } & \multicolumn{2}{|c|}{$\begin{array}{l}\text { Earthworm avoidance test: } \\
\text { avoidance [\%] by highest } \\
\text { test concentration ( } 100 \% \\
\text { soil) }\end{array}$} & \multicolumn{2}{|c|}{$\begin{array}{l}\text { Daphnids: } \\
\text { immobilization [\%] } \\
\text { in } 25 \% \text { soil eluate } \\
\left(0.01 \mathrm{M} \mathrm{CaCl}_{2}\right)\end{array}$} \\
\hline & Test start & Test end & Test start & Test end \\
\hline \multicolumn{5}{|c|}{ Cypermethrin } \\
\hline $01-\mathrm{A}$ & 94 & 72 & 60 & 90 \\
\hline O2-A & 84 & 60 & 15 & 50 \\
\hline $03-G$ & 92 & 96 & 80 & 90 \\
\hline \multicolumn{5}{|c|}{ Carbendazim } \\
\hline $01-A$ & 20 & 8 & 100 & 0 \\
\hline $02-A$ & 4 & 0 & 100 & 5 \\
\hline $03-G$ & 28 & 4 & 100 & 5 \\
\hline
\end{tabular}

substances was unclear and non-reproducible in the previous ${ }^{14} \mathrm{C}$-testing.

\section{Application of a simplified testing tool}

Because the fate and NER-formation of TNT could be explained quite well and a large amount of NER was formed in the ${ }^{14} \mathrm{C}$-tests with this substance, non-labelled TNT was used for further development of the testing tool (see "Experiments with soil for subsequent ecotoxicological testing"). In the radiolabelled experiments, it was found that the rate of mineralization was low and could be ignored. It was further shown that after aging not all extracted radioactivity could be attributed to parent TNT, which could lead to an overestimation of NER while working with non-radiolabelled TNT. As stated in "Development of a testing tool", all uncertainties have to be considered when interpreting the results of the experiment. After 5 months incubation the extractable amount of parent TNT was low. From the ${ }^{14} \mathrm{C}$-experiments it can be concluded that in the three soils most of the added TNT that was missing after 5 months was converted to NER. Thus, NER shown in the following figures were calculated as amount TNT added minus amount TNT extracted.

The results presented in Fig. 5 are comparable for the three soils. NER, residue and potentially bioavailable fractions are expressed as percentage of added TNT.

Aliquots of the soil were extracted with $0.01 \mathrm{CaCl}_{2}$, which gives the actual bioavailable fraction expressed in $\mathrm{mg} / \mathrm{l}$. Further aliquots of the soil were extracted with acetonitrile before and after Tenax extraction $(20 \mathrm{~h})$. The difference of TNT in both acetonitrile extracts should represent the amount of TNT that remains on the Tenax and is defined as potentially bioavailable. The amount of TNT extractable with acetonitrile after Tenax extraction is defined as residue (see detailed definitions in "Development of a testing tool"). NER is defined as the amount of 

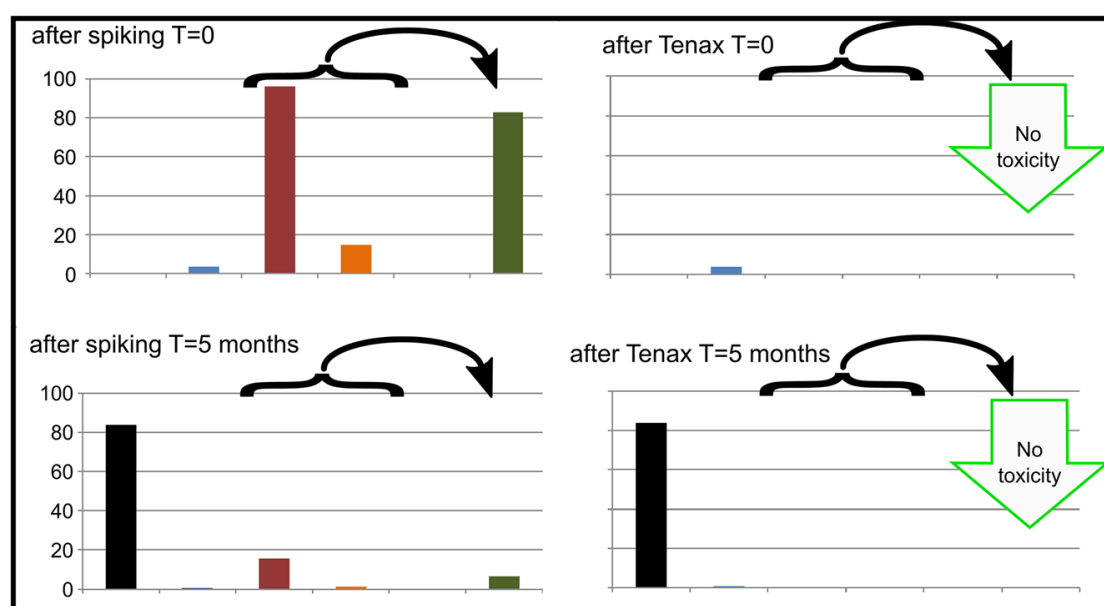

a
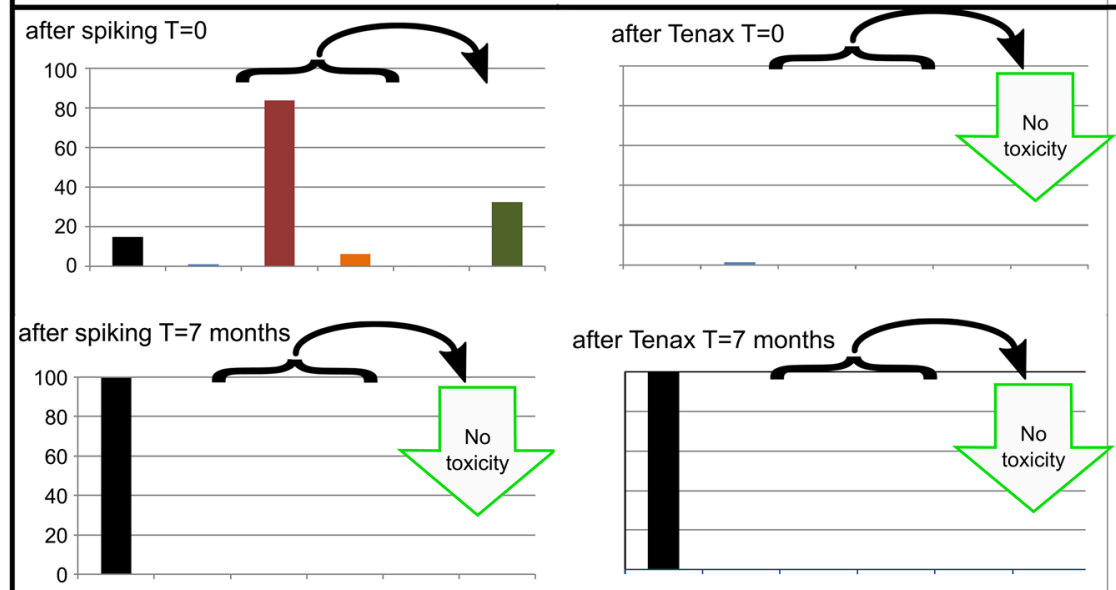

b

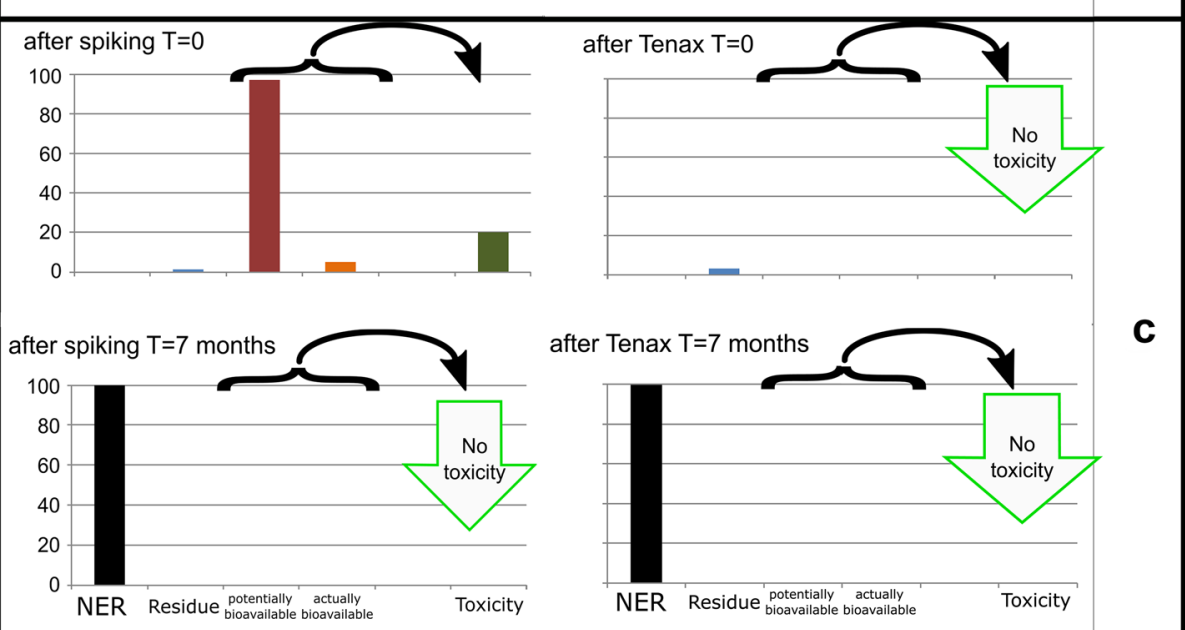

Fig. 5 Application of the testing tool with TNT in 3 different soils. NER, residue and potentially bioavailable expressed as percentage of added TNT, actually bioavailable as mg/ITNT and toxicity as 100/EC20. Potentially bioavailable is calculated as difference of TNT present in soil before and after TENAX-extraction. The same Y-axis is used for all parameters. a Soil 01-A (loamy sand). b Soil 02-A (silt loam). c Soil 03-G (silt loam) 
TNT added minus the sum of TNT extracted as it cannot be measured directly without radiolabelled test substance. The term toxicity is represented by the reciprocal of an effect concentration, in our case inhibition of luminescence, i.e. by 100/EC20.

After spiking (upper left figures in Fig. 5a-c), the toxicity measured by the luminescence bacterial test can be explained by the bioavailable TNT in the freshly spiked soil. After removal of the bioavailable TNT (upper right figures), the same toxicity test shows no toxicity in any of the soils any more.

Aging of the spiked soils for 5 and 7 months, respectively, leads to an increase of NER (lower left figures in Fig. $5 \mathrm{a}-\mathrm{c}$ ). In soil 01-A a small bioavailable fraction remains, coinciding with some measured effects in the luminescence test. In the other two soils, TNT is almost entirely transformed into NER and no toxicity was observed. Application of Tenax removed the bioavailable fraction and the observed toxicity in soil $01 \mathrm{~A}$ (Fig. 5a-c, lower right figures), and no toxicity using luminescence inhibition was observed in any of the soils.

In summary, it was observed that with decreasing bioavailable TNT in the soils the toxicity measured as luminescence inhibition decreased as well. On the other hand, the observed increase of the NER fraction does not coincide with an increase of luminescence inhibition, indicating that NER cannot be responsible for the measured toxicity.

\section{Simplified test tool and implementation in risk assessment} In the experiments described above, different extraction steps are used to quantify fractions as described in Figure 1 by Ortega-Calvo et al. [26]. It was concluded by Ortega-Calvo et al. [26], that methods to be used in risk assessment should be clear for risk assessors and that measurable data have to be provided. Methodologies to understand the fate of chemicals in soils have been established and understood for quite some time in terms of regulation.

However, the assessment of the remaining NER fraction is still missing. In the approach described above, we show a way to measure toxicity of NER after removal of the bioavailable fractions of a chemical from soil. This was not possible until now since previous extraction methods used so far required organic solvents which will damage the soil microflora irreversibly and prevent further ecotoxicological testing of the extraction residues. Using Tenax, in a three-phase system with soil and water, makes it possible to recover soil containing NER, but without the bioavailable fraction of a chemical. Thus, in a further step the ecotoxicity of NER formed from a chemical in soil can be measured without interferences of the still bioavailable fraction of that chemical which is a huge step forward in NER assessment.

However, this is only possible if the fate of the chemical in soils is understood in detail or if radiolabelled test chemicals are used for testing. Measurement of the nonradiolabelled parent might miss significant fate processes and as our experiments have shown, huge differences may occur if in a non-radiolabelled experiment the principle NER $=100 \%$ minus extractable parent is applied. Without detailed information on the fate of a chemical in soils, NER determination without radiolabelled test substance is very unreliable.

The procedure developed in this project has been demonstrated to work well for TNT, but for as yet unclear reasons it was not possible to reproducibly measure the fate of cypermethrin and carbendazim in soil. Understanding the fate of a chemical is essential. However, the procedure is still too complex to be used in a routine risk assessment tool under, e.g. REACH. Nevertheless, it does provide an option to establish the toxicity of NER which might be applied in detailed more in depth risk assessment in special cases for important chemicals. An example of this is environmental testing of pesticides with a ${ }^{14} \mathrm{C}$-radiolabelled test substance. This is mandatory and the procedure described above could easily be applied in the case where relevant NER formation is already known available and the substance shows significant toxicity in ecotoxicological testing.

\section{Conclusions}

Regulations ask for unequivocal and measurable parameters for the quantification of the risks of chemicals, which also applies for NER. To describe the presence of a chemical, we adapted the approach of [26], whose terminology is generally accepted and also described in ISOstandards. The extractable fraction of a chemical contains a bioavailable part, and our hypothesis was that this bioavailable part is responsible for ecotoxicological effects. The actual bioavailable part can be measured by an aqueous extraction and the potential bioavailable part by a recently standardized method using Tenax [20]. Because Tenax can be removed easily from soil, the method can also be applied in order to produce a soil without a bioavailable fraction of a chemical. Consequently, when using this method the soil remains suitable for the application of most ecotoxicological tests to prove our hypothesis.

We studied three NER-forming chemicals and followed their fate for a period of 6 months after spiking in three different soils. In experiments using ${ }^{14} \mathrm{C}$ radiolabelled chemicals, NER could be measured directly. In these experiments formation of NER was observed for all chemicals tested. 
For 2,4,6-trinitrotoluene (TNT), NER-formation was reproducible. By removing the bioavailable fractions directly after spiking and after aging it was possible to remove toxicity. The experiments with and without ${ }^{14} \mathrm{C}$-radiolabelled TNT clearly showed that toxicity was caused by the bioavailable chemical and not by NER.

With the other two selected chemicals, cypermethrin and carbendazim, results were less clear because there was a large uncertainty about their fate. The degree of biodegradation was not reproducible for cypermethrin and unexpected and considerable losses occurred with carbendazim during the experiments, which could not be explained by the experimental setup. This resulted in a very large uncertainty about NER-formation in experiments using non-radiolabelled chemicals. Thus, it is not possible to draw conclusions from non-radiolabelled experiments with these chemicals.

\section{Discussion}

In summary, we developed a tool that can be used to measure NER toxicity, provided that the fate of the chemical, including NER formation, is understood and reproducible. Additional experiments using radiolabelled chemicals remain necessary if uncertainties about the fate of chemicals exist. The tool is still too complex to be used in routine risk assessment, but it provides an option to directly measure NER toxicity in soil degradation testing of chemicals.

Our experiments confirmed that for the determination of NER in soil radiolabelled chemicals are necessary. NER in soil cannot be measured directly with conventional chemical analysis without radiolabelling. However, even using ${ }^{14} \mathrm{C}$-radiolabelled compounds uncertainty might remain about the fate of a substance. The actual NER determination based on radiolabelling only quantifies NER, but does not provide information on the nature of the NER measured. An approach for chemical characterization of NER for regulation was recently published [22] and further projects are currently being conducted to develop tools for regulatory use. Nevertheless, this new tool might provide an improved exposure assessment. Data about potential effects of NER, which are required for a risk assessment of NER, are still missing. Our project provides an option of how to determine the ecotoxicological potential of NER.

NER is not measurable with non-radiolabelled chemicals and will remain the subject of discussion. In both tools, the characterization of NER and the determination of toxicity of NER are very important in order to get a better understanding of NER based on scientific findings. This might help the development of a science-based risk assessment of NER in the future that can replace the pragmatic but scientifically ungrounded approach, which is followed in current regulation.

\section{Abbreviations}

AR: applied radioactivity; ${ }^{\circ} \mathrm{C}$ : degrees celsius; dm: dry matter; h: hour; kBq: kiloBecquerel; LSC: liquid scintillation counting; mg/kg: milligram per kilo; mg/l: milligram per liter; NER: non-extractable residues; $\mathrm{PAH}$ : polycyclic aromatic hydrocarbons; PLE: pressurized liquid extraction; TLC: thin layer chromatography; TNT: 2,4,6-trinitrotoluene.

\section{Acknowledgements}

This paper is based on a project initiated and financed by CEFIC LRI (Brussels) as 'Development of Soup Tests for the Risk Assessment of NER in Soil. LRI-ECO25'.

\section{Authors' contributions}

All authors contributed to the study conception and design. Material preparation, data collection and analysis were performed by KHR, JD, JH, DH and JL. The first draft of the manuscript was written by $\mathrm{JH}$ and all authors commented on previous versions of the manuscript. All authors read and approved the final manuscript.

Funding

This study was funded by CEFIC LRI (Brussels). Grant number LRI-ECO25.

Availability of data and materials

Not applicable.

Ethics approval and consent to participate

Not applicable.

Consent for publication

Informed consent was obtained from all individual participants included in the study.

\section{Competing interests}

The authors declare that they have no competing interests.

\section{Author details}

${ }^{1}$ Wageningen Environmental Research, P.O.Box 47,6700 AA Wageningen, The Netherlands. ${ }^{2}$ Fraunhofer Institute for Molecular Biology and Applied Ecology, Auf dem Aberg 1, 57392 Schmallenberg, Germany.

Received: 15 October 2019 Accepted: 16 December 2019

Published online: 27 December 2019

\section{References}

1. Barriuso E, Benoit P, Dubus I (2008) Formation of pesticide nonextractable (bound) residues in soil: magnitude, controlling factors and reversibility. Environ Sci Technol 42:1845-1854

2. Cornelissen G, van Noort PCM, Govers HAJ et al (1997) Desorption kinetics of chlorobenzenes, polycyclic aromatic hydrocarbons, and polychlorinated biphenyls: sediment extraction with tenax and effects of contact time and solute hydrophobicity. Environ Toxicol Chem 16(7):1351-1357

3. ECETOC (2012a) Understanding the relationship between extraction technique and bioavailability. ECETOC Technical Report 117, Brussels

4. ECETOC (2012b) Development of interim guidance for the inclusion of non-extractable residues (NER) in the risk assessment of chemicals. ECETOC Technical Report 118, Brussels

5. ECHA_2017_R.11. Guidance on Information Requirements and Chemical Safety Assessment, Chapter R.11: Endpoint specific guidance (PBT/VPVB assessment), version 3.0, June 2017

6. ECHA_2017_R.7b. Guidance on Information Requirements and Chemical Safety Assessment, Chapter R.7b: Endpoint specific guidance, version 4.0, June 2017 
7. ECHA_2017_R.7c. Guidance on Information Requirements and Chemical Safety Assessment, Chapter R.7c: Endpoint specific guidance, version 3.0, June 2017

8. EFSA (2015) Statement on the FERA guidance proposal: 'Guidance on how aged sorption studies for pesticides should be conducted, analysed and used in regulatory assessments' (FERA, 2012). EFSA J 13(7):4175

9. Eschenbach A, Oing K (2013) Erarbeitung eines gestuften Extraktionsverfahrens zur Bewertung gebundener Rückstände. Expert opinion for UBA, FKZ 36001070 2013: 101

10. FERA (2012) Statement on the FERA guidance proposal: 'Guidance on how aged sorption studies for pesticides should be conducted, analysed and used in regulatory assessments'

11. Harmsen J, Frintrop P (2003) Non-halogenated and volatile compounds. In: Thompson KC, Nathanail P (eds) Chemical analysis of contaminated land. Sheffield analytical chemistry, vol 6. Blackwell Publishing, Hoboken, pp 189-215

12. Harmsen J, Hennecke D, Hund-Rinke K, Lahr J, Deneer J (2018) Advances in the development of procedures to establish the toxicity of nonextractable residues (NER) in soil. LRI-ECO25. Wageningen, Wageningen Environmental Research, Report 2909. Fraunhofer IME report 040215996.114 pp. http://edepot.wur.nl/463765

13. Harmsen J, Rietra R (2018) 25 years monitoring of PAHs and petroleum hydrocarbons biodegradation in soil. Chemosphere 207:229-238

14. Houba VJG, Temminghoff EJM, Gaikhorst GA, van Vark W (2000) Soil analysis procedures using $0.01 \mathrm{M}$ calcium chloride as extraction reagent. Commun Soil Sci Plant Anal 31:9-10, 1299-1396

15. ISO 11348 (2007) Water quality-determination of the inhibitory effect of water samples on the light emission of Vibrio fischeri (Luminescent bacteria test). ISO, Geneva

16. ISO 15685 (2004) Soil quality-Determination of potential nitrification and inhibition of nitrification-Rapid test by ammonium oxidation. ISO, Geneva

17. ISO 11916-1 (2013) Soil quality-determination of selected explosive compounds-Part 1: Method using high-performance liquid chromatography (HPLC) with ultraviolet (UV) detection. ISO, Geneva

18. ISO 17402 (2008) Soil quality_-Requirements and guidance for the selection and application of methods for the assessment of bioavailability of contaminants in soil and soil materials. ISO, Geneva

19. ISO 17512-1 (2008) Soil quality-Avoidance test for determining the quality of soils and effects of chemicals on behaviour-Part 1: test with earthworms (Eisenia fetida and Eisenia andrei). ISO, Geneva

20. ISO/TS 16751 (2018) Soil quality —environmental availability of non-polar organic compounds - determination of the potential bioavailable fraction and the non-bioavailable fraction using a strong adsorbent or complexing agent. ISO, Geneva

21. Joos A, Knackmuss H-J, Spyra W (2008) Leitfaden-Natürliche Schadstoffminderung bei sprengstofftypischen Verbindungen. BMBF-Förderschwerpunkt KORA, Themenverbund 5 Rüstungsaltlasten. IABG mbH (Hrsg), Berlin, ISBN 978-3-00-025181-8

22. Kästner M, Trapp A, Schäffer A (2018) Consultancy services to support ECHA in improving the interpretation of Non-Extractable Residues (NER) in degradation assessment. Discussion paper-final report. Publisher: European Chemical Agency ECHA, July 2018. https://www.echa.europ a.eu/documents/10162/13630/echa_discussion_paper_en.pdf/4185c f64-8333-fad2-8ddb-85c09a560f7c
23. Katan J, Fuhremann W, Lichtenstein EP (1976) Binding of 14C parathion in soil: a reassessment of pesticide persistency. Science 193:891-894

24. Matthies M, Glinka K, Theiling M, Hideg K, Steinhoff HJ (2016) Kinetics of rapid covalent bond formation of aniline with humic acid: ESR investigations with nitroxide spin labels. Appl Magn Reson 47:627-641

25. OECD Test Guideline 202 (2004) Daphnia sp. acute immobilisation test. https://www.oecd-ilibrary.org/environment/test-no-202-daphnia-spacute-immobilisation-test_9789264069947-en

26. Ortega-Calvo JJ, Harmsen J, Parsons JR, Semple KT, Aitken MD, Ajao C, Eadsforth C, Galay-Burgos M, Naidu R, Oliver R, Peijnenburg WJGM, Römbke J, Streck G, Versonnen B (2015) From bioavailability science to regulation of organic chemicals. Environ Sci Technol 2015(49):10255-10264

27. Reichenberg F, Mayer P (2006) Two complementary sides of bioavailability: Accessibility and chemical activity of organic contaminants in sediments and soils. Environ Toxicol Chem. 25:1239-1245

28. Schäffer A, Kästner M, Trapp S (2018) A unified approach for including non-extractable residues (NER) of chemicals and pesticides in the assessment of persistence. Environ Sci Pollut Res 30:51

29. Trapp S, Brock AL, Nowak K, Kästner M (2018) Prediction of the formation of biogenic nonextractable residues during degradation of environmental chemicals from biomass yields. Environ Sci Technol 52:663-672

30. Umeh AC, Duan L, Naidu R, Semple KT (2018) Enhanced recovery of nonextractable benzo[a]pyrene residues in contrasting soils using exhaustive methanolic and nonmethanolic alkaline treatments. Anal Chem 90(21):13104-13111

31. Wais A (1998) Non-extractable residues of organic xenobiotics in soils-a review. In: Führ F, Ophoff H (eds) Pesticide bound residues in soil. Wiley, Weinheim, pp 5-31

32. Wang YF, Xu J, Shan J, Ma YN, Ji R (2017) Fate of phenanthrene and mineralization of its non-extractable residues in an oxic soil. Environ Pollut 224:377-383

33. Zhu X, Dsikowitzky L, Kucher S, Ricking M, Schwarzbauer J (2019) Formation and fate of point-source nonextractable DDT-related compounds on their environmental aquatic-terrestrial pathway. Environ Sci Technol 53:1305-1314

34. Zhu X, Schroll R, Dörfler U, Chen B (2017) Inoculation of soil with an isoproturon degrading microbial community reduced the pool of "real nonextractable" isoproturon residues. Ecotoxicol Environ Saf 149:182-189

\section{Publisher's Note}

Springer Nature remains neutral with regard to jurisdictional claims in published maps and institutional affiliations.

\section{Submit your manuscript to a SpringerOpen ${ }^{\circ}$ journal and benefit from:}

- Convenient online submission

- Rigorous peer review

- Open access: articles freely available online

- High visibility within the field

- Retaining the copyright to your article

Submit your next manuscript at springeropen.com 